\title{
COMPETENCIAS INVESTIGATIVAS EN LOS DOCENTES BENEFICIADOS POR LA ESTRATEGIA DE FORMACIÓN Y ACCESO PARA LA APROPIACIÓN PEDAGÓGICA DE LAS TIC
}

\author{
Por: Luis Eduardo Paz Saavedra ${ }^{1}$ - Yicela del Pilar Fierro Marcillo ${ }^{2}$
}

\section{RESUMEN}

El presente artículo recoge los resultados de la investigación realizada en los departamentos de Cauca, Nariño y Putumayo, luego de la formación ofrecida por el programa Computadores para Educar y la Universidad de Nariño en el diplomado "Apropiación Pedagógica de las TIC". El estudio aborda el fortalecimiento de competencias investigativas luego de dicha formación y del desarrollo de proyectos pedagógicos de aula en los que se pretende la solución de problemáticas propias del contexto de profesores y estudiantes. Entre los aspectos analizados se destaca la actitud que tienen los docentes frente al papel de las TIC en los procesos investigativos, la forma en que estas tecnologías contribuyen a actividades investigativas y a competencias específicas que se ven fortalecidas en este campo. Adicionalmente se analizan los factores que tienen incidencia en todo el proceso, las oportunidades y dificultades a tenerse en cuenta en busca del fortalecimiento de dichas competencias, así como los elementos relevantes cuando se trata de realizar la integración de TIC en los currículos escolares, a fin de mejorar las posibilidades de desarrollo de la investigación escolar.

Palabras clave: Educación, profesores, competencias, política educativa.

Clasificación JEL: 112, 128.

1. Magister en informática Educativa, Máster en Tecnología Multimedia Licenciado en Informática, Especialista en Docencia Universitaria, Docente tiempo Completo Universidad de Nariño. Director proyecto Computadores para Educar - UDENAR. Correo electrónico: luisepaz@gmail.com

2. Especialista en Gerencia Informática Licenciada en Informática, Profesional en Pedagogía proyecto Computadores para Educar, Universidad de Nariño. Correo electrónico: giselaonline08@gmail. com

Artículo recibido: 11 de noviembre de 2014.

Aprobación definitiva: 5 de febrero de 2015. 


\title{
INVESTIGATIVE SKILLS IN TEACHERS BENEFITED BY THE TRAINING STRATEGY FOR EDUCATIONAL ACCESS AND APPROPRIATION OF THE IT
}

\author{
By: Luis Eduardo Paz Saavedra Yicela del Pilar Fierro Marcillo
}

\begin{abstract}
This paper presents the results of a research conducted in the departments of Cauca, Nariño and Putumayo, after the completion of a training program offered by Computadores para Educar and the University of Nariño in the course "Pedagogical Appropriation of the IT". This study approaches the improvement of investigative skills after the completion of the mentioned training and the development of classroom teaching projects in which it's expected to find a solution for issues relevant from a teachers and students point of view. Among the analyzed aspects, are highlighted the attitude that teachers have towards the role of the IT in the research process, how these technologies contribute to research activities and specific skills that are strengthened in this field. Additionally, the factors that impact the process are discussed; likewise, the opportunities and challenges to be considered in the seek to strengthen such skills as well as relevant elements when it comes to integrating the IT into school curriculums in order to improve the chances of scholar research development.
\end{abstract}

Keywords: Education, teachers, skills, education politics.

JEL Classification: I12, I28. 


\section{Introducción}

La influencia de las tecnologías de la información y la comunicación (TIC) en la sociedad es incuestionable, y hoy en día estas herramientas se han constituido en partes fundamentales de la cultura tecnológica que nos rodea y con la cual debemos convivir. Así, el inmenso valor otorgado a las TIC, exige que también los sistemas educativos a nivel mundial implementen propuestas educativas acordes a las exigencias de la vida moderna, con las cuales se pueda hacer frente a la necesidad de incorporar dicha tecnología en favor de los procesos de enseñanza y aprendizaje.

Frente al anterior panorama, el Ministerio de Educación Nacional de Colombia y el Ministerio de Tecnologías de la Información y la Comunicación, a partir del planteamiento del Plan Nacional de Tecnologías de la Información y las Comunicaciones (MinTIC, 2008), han propuesto el desarrollo de diferentes líneas de acción que apuntan a fomentar la utilización y apropiación de las TIC en el sector educativo mediante el impulso de proyectos de inversión en tecnología, dotación de infraestructura tecnológica, formación docente, desarrollo de competencias para el uso de TIC por parte de estudiantes, articulación con las políticas de uso e incorporación de herramientas tecnológicas dentro del campo de formación en el nivel profesional, entre otros.

Ante las iniciativas planteadas, es importante llevar a cabo un análisis de las diferentes propuestas que se han puesto en marcha dentro del campo educativo, esto teniendo en cuenta las actividades propias de los establecimientos educativos y el impacto generado dentro de un contexto estrechamente ligado a las iniciativas que realizan los sectores gubernamentales; en este caso, el análisis realizado se refiere a los aportes realizados por la "estrategia de formación y acceso para la apropiación pedagógica de las TIC” (CPE, 2014, p. 3), impulsada en los últimos años por parte de Computadores para Educar.

La estrategia se centra en el proceso de dotación de equipos y capacitación docente en el campo de la apropiación pedagógica de las TIC. Esta formación incluye un alto componente en competencias en siete dimensiones: disciplinares, pedagógicas, actitudinales, comunicativas, técnicas y tecnológicas, evaluativas e investigativas; de esta forma se contribuye, directa e indirectamente, a cumplir con los requerimientos de formación contemporáneos, los cuales se encaminan a otorgar un gran valor a las personas con habilidades de autonomía, competitividad, autoaprendizaje y adaptabilidad; es decir, personas que de acuerdo a las nuevas tendencias pedagógicas, puedan definirse como competentes (Gonzáles \& Sánchez, 2003).

La inclusión de las competencias en el contexto educativo surge de la necesidad de dar un enfoque curricular centrado en los requerimientos sociales modernos, centrados en una aplicación creativa, responsable y eficiente de conocimientos, habilidades y actitudes: "aprender a conocer, aprender a hacer, aprender a convivir se convierte en tres pilares de la educación para hacer frente a los retos del siglo XXI y llevar a cada persona a descubrir y despertar e incrementar sus posibilidades creativas" (Delors, 1996, p. 16). 
Respecto a la importancia de las competencias, los conocedores y sus estudios realizados hacen diversos aportes. Carlos González Díaz y Leonardo Sánchez Santos, por ejemplo, identifican a las competencias básicas como aquellas en las que la persona construye las bases de su aprendizaje (interpretar y comunicar información, razonar creativamente y solucionar problemas, entre otras), y que reafirman la noción del aprendizaje continuado y la necesidad de aprender a aprender (Gonzales \& Sánchez, 2003).

Las competencias también se entienden como actuaciones integrales para identificar, interpretar, argumentar y resolver problemas del contexto con idoneidad y ética, integrando el saber ser, el saber hacer y el saber conocer (Muñoz, Quintero \& Múnevar, 2001). Esto demuestra la importancia de una formación que permita al profesor realizar una aplicación de las mismas en el entorno inmediato de acción, respondiendo a los retos que impone la modernización, los cuales en su mayoría desembocan en la necesidad del fortalecimiento de los procesos de enseñanza y aprendizaje, desarrollando no únicamente aptitudes cognitivas, sino también actitudes y destrezas orientadas a las necesidades y soluciones que se requieren.

Si bien a través de la formación en apropiación pedagógica de las TIC se busca fortalecer los siete tipos de competencias mencionados, en el caso de las competencias investigativas, Muñoz et al. (2001) las identifican como indispensables para que el educador se constituya como investigador por excelencia. De ésta forma las competencias investigativas se introducen en el campo educativo como parte integral del quehacer docente ya que le permiten fortalecer conocimientos y habilidades, y aplicarlos en situaciones reales con mediación de la tecnología. A propósito, Cendales y Mariño señalan que la Investigación resulta definitivamente ligada a los compromisos éticos y políticos del maestro, a la acción, a la transformación de lo cotidiano; inclusive, van más allá de esta reflexión al expresar poéticamente, que investigar es un acto de compromiso con la historia, asumiendo un lugar en ella (Cendales \& Mariño, 2003).

Para los docentes de las sedes beneficiadas por la estrategia de CPE se tiene un esquema en el cual el profesor puede fortalecer sus competencias investigativas y aprovechar éstas habilidades para fomentarlas en sus estudiantes; situación que se procura lograr a través de la construcción de un proyecto pedagógico de aula en TIC. Éste elemento permitirá a su vez, generar conocimiento desde la praxis en el entorno inmediato y con una mirada propia que refleja objetivamente la realidad para encontrar una solución acorde con ella. En éste sentido, la estrategia de formación aborda la dimensión investigativa durante cinco momentos puntuales:

1. Inicialmente, el docente se sumerge en el trabajo investigativo sobre los aportes de la formación en TIC y su contribución pedagógica.

2. Las iniciativas en investigación parten de una pregunta. Éste cuestionamiento es el que orientará el desarrollo de todo el proyecto, pues su fin último es dar solución a tal inquietud.

3. El docente explora, analiza, compara y elige la opción adecuada en una metodología a seguir para la ejecución de su proyecto de aula en TIC. 
4. El éxito del proyecto investigativo, depende en gran medida de los resultados de las acciones realizadas, las cuales deben ser evaluadas tanto en los resultados del proyecto, como en la evolución de la mejoría de los aprendizajes.

5. El proceso final se consolida con la demostración de los logros obtenidos, evidenciando cada uno de los pasos abordados, el proceso desarrollado y el resultado final en la transformación educativa obtenida.

Con base en las anteriores consideraciones, y con el fin de reconocer la labor investigativa de los profesores beneficiados con la formación descrita, este artículo se centra en analizar el grado de influencia que tiene la estrategia de formación sobre el fortalecimiento de las competencias investigativas en los docentes de los departamentos de Nariño, Cauca y Putumayo, durante el proceso de planteamiento, ejecución y evaluación de su proyecto pedagógico de aula. Para lograrlo, es preciso reconocer las competencias investigativas y las herramientas TIC que ejercen mayor influencia en este campo, así como la percepción del docente frente a la inclusión tecnológica en la investigación educativa; todo ello mediado por la existencia de recursos tecnológicos y las condiciones sociales, económicas, culturales, entre otras que rodean a los maestros en el ejercicio de su práctica in situ.

Como se podrá observar en los resultados que se describen más adelante, la aplicación de la tecnología aporta enormemente al proceso de construcción y reconstrucción del conocimiento, lo cual permite ofrecer una amplia gama de alternativas de solución a los problemas de los estudiantes, del proceso de enseñanza aprendizaje, de la comunidad, del entorno local y regional. De ésta forma, las competencias investigativas con inclusión de TIC podrán estar definidas dentro del proyecto educativo institucional integrando las diferentes áreas y disciplinas, con un proceso que inicia en la formación docente y finaliza en el mejoramiento de los procesos educativos llevados a cabo.

\section{Metodología}

La estrategia de formación y acceso para la apropiación pedagógica de las TIC busca, entre otros aspectos, fomentar en los docentes el desarrollo de ciertas habilidades relacionadas con el área investigativa; de hecho, éstas son explicitadas cuando se construye el proyecto de aula. Con relación a ello, la investigación se enfocó en los docentes pertenecientes a sedes tipo A y F, de acuerdo con la clasificación dada por Computadores para Educar (CPE, 2012). Para éste caso, las sedes tipo A corresponden a aquellas instituciones educativas beneficiadas por primera vez, tanto en donación de equipos como en formación. Por su parte las sedes de tipo F recibieron los equipos de cómputo anteriormente, sin embargo, los docentes no habían obtenido la formación correspondiente, razón por la cual fueron convocados con el fin de realizar un proceso educativo completo.

Los docentes que hacen parte de la población objeto de estudio, presentan unas características muy específicas relacionadas con el lugar en el que realizan su labor educativa, si se parte de que la Región 6 (Cauca, Nariño y Putumayo) es una zona que presenta innumerables condiciones de diversos tipos, que sin duda alguna afectan la labor del docente; por ejemplo, las sedes de tipología A se caracterizan 
principalmente por ubicarse en lugares de difícil acceso, condiciones ambientales y climáticas adversas, situaciones de conflicto, dificultades en la energía eléctrica, etc. Respecto a las sedes tipo B, presentas características similares, aunque normalmente hay un mayor conocimiento de su contexto pues ya han sido atendidas anteriormente.

Estas características poblacionales permiten poner en contexto la temática del estudio, orientando al análisis de tres aspectos fundamentales, con sus respectivas variables: uno, las competencias investigativas que se ven mayormente fortalecidas con la formación durante los distintos momentos del proceso investigativo; dos, las herramientas TIC que por su inclusión efectiva están realizando un aporte significativo en el fortalecimiento de competencias; tres, la percepción del maestro en cuanto a las herramientas TIC y su papel definitivo en la investigación.

Todo éste proceso se desarrolla a partir de un enfoque mixto de investigación, caracterizado por la comprensión e interpretación de hechos y fenómenos sociales. Desde el enfoque cualitativo, Taylor \& Bogdan (1989) consideran esta alternativa tipo como "aquella que produce datos descriptivos: las propias palabras de las personas, habladas o escritas, y la conducta observable” (p. 20). En efecto, la investigación toma como base las producciones propias de los docentes, su actuación en la resolución de problemas del entorno y la implementación de las TIC como mediadoras para el logro de los objetivos en competencias investigativas. Estos resultados solo pueden ser descritos desde la sensibilidad que genera el trabajo desarrollado en campo. Para complementar el anterior enfoque, es necesario puntualizar que también se hace necesaria la recolección de datos que demandan un tratamiento cuantitativo a fin de obtener datos específicos que ameritan un análisis estadístico.

\section{Resultados}

Para el desarrollo de esta investigación se contó con una muestra de 525 docentes, sobre un total de 6421 capacitados dentro de la estrategia nacional de formación y acceso para la apropiación pedagógica de las TIC en los departamentos de Cauca, Nariño y Putumayo, durante los años 2012 y 2013. El tamaño de la muestra seleccionada se estableció con un nivel de confianza del 95\% y un error muestral de 4.1\%. Su selección se realizó por medio de un sistema de muestreo probabilístico y sistemático, de acuerdo con los listados de docentes formados proporcionados por la coordinación pedagógica del proyecto. La recopilación de información se realizó a través de encuestas aplicadas directamente con los docentes participantes y su diligenciamiento fue apoyado por el gestor TIC responsable del acompañamiento durante el proceso formativo de cada docente.

Con base en dicha muestra, se estableció que el 55 \% de los docentes son mujeres y el $45 \%$ hombres. Sus edades oscilan entre 20 y 65 años, siendo las más frecuentes las comprendidas entre 30 y 39 años (32\%) y 40 y 49 años $(32,5 \%)$.

1. Conocimientos en TIC. Con relación a este tema, se analizaron las herramientas trabajadas en el proceso formativo, de las cuales se evaluaron dos aspectos: los conocimientos de los docentes en el uso de dichas herramientas para sus actividades personales y los conocimientos en su uso para el apoyo a sus clases. 
Respecto al uso personal el promedio de la calificación de los docentes es de 3,6 en una escala de 1 a 5 . Todas las valoraciones en el uso de herramientas como programas ofimáticos y servicios de internet están por encima de 3,0 excepto el uso de software de autor con una evaluación de 2,9. Respecto al uso educativo, las evaluaciones son levemente menores en todos los aspectos (promedio de 3,5), situación que claramente indica que, si bien los docentes tienen buenos conocimientos en el uso de estas herramientas, no se sienten plenamente preparados para su incorporación como herramientas de apoyo en el desarrollo de sus clases.

Fig. 1. Calificación de los docentes en el uso de TIC para actividades personales y para su uso en clase

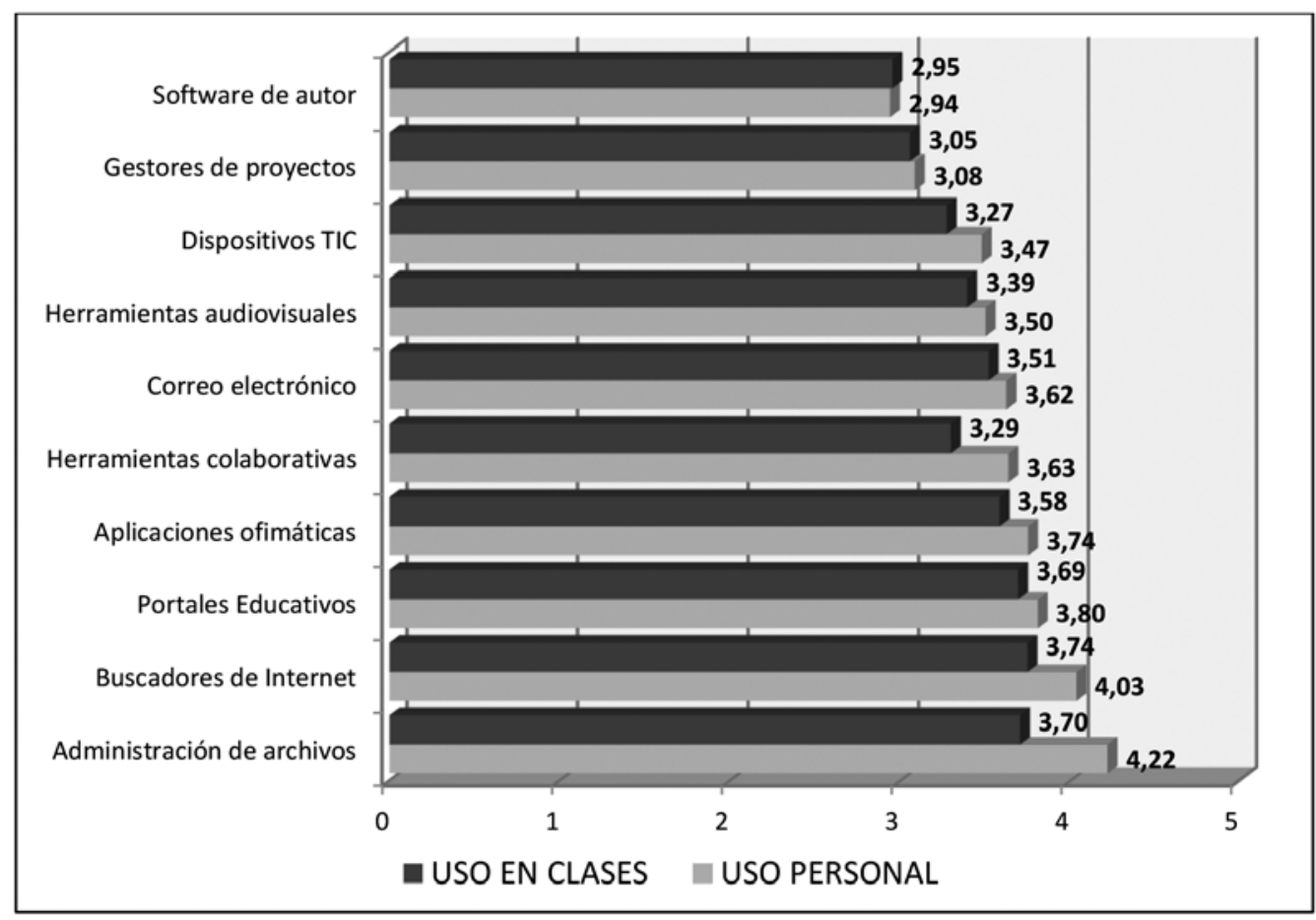

Fuente: Esta investigación

Como se puede observar, los buscadores de internet y herramientas de administración de archivos se destacan como los servicios con la mejor valoración en cuanto al conocimiento por parte de los docentes tanto en el uso a nivel personal como laboral. Este dato es de gran importancia si se tiene en cuenta que el uso de internet y de otros servicios informáticos en la investigación, se constituyen en la principal fuente de información y por tanto, la principal herramienta de trabajo para el investigador. 
A nivel escolar, de la mano de internet y las herramientas web 2.0, se encuentran los portales educativos (3,80 en la evaluación personal y 3,69 en el uso en clases), espacios web dedicados a ofrecer múltiples servicios a la comunidad educativa en cuanto a información, instrumentos de búsqueda, recursos educativos, formación, asesoría, entretenimiento, entre otros, los cuales contribuyen a que internet se convierta en un medio que brinda la oportunidad de equiparar las posibilidades de acceso al saber a todas las comunidades.

2. Investigación pedagógica y TIC. Como ya se explicó, uno de los propósitos fundamentales de la estrategia es el desarrollo de competencias investigativas en los docentes, de manera que puedan replicar dichas competencias con sus estudiantes y fomentar su espíritu investigativo. Respecto a este tema, la primera parte del análisis tiene que ver con la actitud de los docentes frente a la investigación. A la pregunta ¿usted realiza investigación pedagógica? el 74,5\% de los docentes encuestados afirman que si realizan este tipo de procesos, mientras que el restante $25,5 \%$ afirma que no. Asimismo, la totalidad de profesores consideran que la investigación pedagógica es importante para su actividad profesional, aunque un 2,3\% afirma que no está interesado en desarrollarla.

Por otra parte, frente a la pregunta de si creen que las TIC son útiles para realizar investigación pedagógica, casi la totalidad de docentes considera que sí (99\%) mientras menos del 1\% de los profesores (solo 4, de un total de 525 encuestados) afirmaron que no, pues consideran que no es posible usarlas dentro de su contexto específico, dado que pertenecen a lugares muy alejados con un acceso casi nulo a la tecnología.

El docente da un reconocimiento claro y especial a la investigación pedagógica y la considera vital en el campo educativo, no obstante, los docentes en su quehacer diario se ven enfrentados a múltiples situaciones que van desde el qué y el cómo enseñar, hasta la comprensión de las inquietudes que se generan en el estudiante. En este caso, los profesores con frecuencia resuelven estas situaciones utilizando la preparación recibida durante sus años de formación; otras, a través de la revisión de la literatura existente o a partir de lo reportado en experiencias de sus colegas. Al decir de Heller este saber es "la suma de nuestros conocimientos sobre la realidad que utilizamos de un modo efectivo en la vida cotidiana del modo más heterogéneo (como guía para las acciones, como temas de conversación, etc.)" (Heller, 1987: 317).

3. Aplicabilidad de las TIC en los procesos investigativos. Para determinar las TIC de mayor y menor influencia en los procesos investigativos, inicialmente se analizaron las herramientas consideradas de mayor aplicabilidad en investigación. Los resultados indican que en términos generales, todas las aplicaciones informáticas que ya fueron señaladas (ver figura 1), son consideradas como muy aplicables $(45,5 \%)$ o aplicables $(43.7 \%)$ en este campo. Entre estas herramientas, las de mayor acogida son las aplicaciones ofimáticas, los dispositivos usados en entornos educativos (proyectores, reproductores de audio y video, equipos informáticos, etc.), y servicios de internet tales como los navegadores, buscadores y correo electrónico.

En cuanto a las TIC de menor aplicación para la investigación pedagógica se encuentran el software de autor, las herramientas para administración de archi- 
vos y los gestores de proyectos, los cuales en promedio son considerados como poco aplicables o inaplicables, por cerca del 15\% de los docentes. Esta cifra revela que existe un pequeño grupo de docentes con un marcado desconocimiento de las posibilidades que ofrecen tales herramientas dentro del campo investigativo, especialmente en las actividades asociadas al desarrollo del proyecto de aula. Por este motivo se considera importante reforzar y profundizar en los aprendizajes del docente, especialmente en la aplicabilidad de los mismos en todas las actividades del proyecto de aula y de la investigación pedagógica en general. En muchos de los casos observados el docente prescinde del uso de éstas herramientas dada la tendencia que tiene a utilizar aquellos elementos que ya conoce, sin arriesgarse a explorar campos que probablemente requerirán mayor dedicación y producción intelectual.

Sobre las herramientas con evaluación más baja, es importante aclarar que las herramientas de autor están vinculadas con la producción colaborativa de saberes desde el profesor hacia la comunidad estudiantil y confluyen en la idea de aprovechar el software educativo para diseñar materiales que estimulen las capacidades de los estudiantes. Este hecho en el quehacer educativo es explicado por Gardner en su teoría de las inteligencias múltiples, en la cual se analiza la necesidad de estimular el talento de cada niño para que él descubra por sí mismo un área de capacidad (Gardner, 1983). A partir de ésas inteligencias existentes el docente puede trabajar las potencialidades del educando transformando el proceso de aprendizaje convencional en un juego provechoso, divertido e interesante, en el que el software de autor puede ser de mucha ayuda.

Por otra parte, los gestores de proyectos y las herramientas colaborativas se constituyen en los principales elementos para compartir la experiencia lograda con los estudiantes y obtener la retroalimentación que el proyecto requiere para avanzar en su consolidación final, transmitiendo las potencialidades y fortalezas a otros contextos; ello asegura que el proyecto trascienda las paredes del aula y sensibilice a toda la comunidad educativa en torno a la generación de nuevas soluciones a las dificultades presentadas en el contexto educativo. Adicionalmente, las herramientas colaborativas fortalecen la creación de vínculos investigativos entre docentes y estudiantes, generando espacios de discusión y negociación a partir de una metodología activa que propende por la interacción social, la capacidad de comunicarse, el sentido de colaboración, el cambio de actitudes, el desarrollo del pensamiento y el descubrimiento de nuevas alternativas de solución a las problemáticas.

4. Frecuencia de uso de TIC en los procesos investigativos. El siguiente aspecto evaluado, además de la aplicabilidad, corresponde a la frecuencia de utilización de herramientas TIC en el desarrollo de actividades relacionadas con la investigación. Al respecto, los resultados indican que el 62\% de los docentes piensan utilizarlas con mucha frecuencia, el 34\% con poca frecuencia y el $4 \%$ no piensan usarlas, situación que de alguna manera se relaciona con la imagen que ese $4 \%$ tiene, en el sentido se considerar que las TIC no son útiles para estas actividades, ya sea por desconocimiento parcial de la herramienta o por apatía frente al tema. Otro factor que incide en este porcentaje de docentes que piensan usarlas con poca frecuencia o que no las usaría, tiene que ver con las dificultades que enfrentan en los lugares 
en los que desarrollan su labor educativa, los cuales aún no cuentan con la infraestructura y los recursos tecnológicos y de acceso necesarios para brindar a los docentes las condiciones necesarias para este tipo de actividades.

Las herramientas y servicios que se considera utilizar con más frecuencia, son los servicios de búsqueda en internet, aplicaciones ofimáticas y servicios que ofrece la Web 2.0, pues el protagonismo otorgado a estas herramientas modifica las actividades de los docentes, para convertirse en un cúmulo de informaciones y oportunidades educativas que transforman los espacios formativos. En palabras de Castells: "El nuevo sistema de comunicación transforma radicalmente el espacio y el tiempo, las dimensiones fundamentales de la vida humana" (Castells, 1997, p. 174).

Pasando a las TIC que tendrían el menor uso en procesos investigativos, similar al caso anterior también se encuentran el software de autor, los gestores de proyectos y herramientas colaborativas. Los resultados sobre estos dos últimos servicios son particularmente interesantes, dado que, precisamente, son aquellos que ofrecen mayores potenciales para el trabajo investigativo; por ejemplo, a partir de las utilidades que tienen las aplicaciones de edición, profesores y estudiantes pueden elaborar fácilmente materiales de manera individual o grupal, compartirlos y someterlos a una evaluación crítica por parte de sus pares. La esencia de estas herramientas se apoya en un componente investigativo del alto impacto y aplicada al ámbito educativo constituye un importante medio para construir el conocimiento de forma colaborativa, mediante aportaciones individuales que enriquecen el aprendizaje y la práctica docente.

5. Competencias investigativas. Como parte de la investigación, se propuso identificar las competencias investigativas que se ven influenciadas en mayor y menor grado a través de la estrategia de formación ofrecida. Para analizar este tema se tuvieron en cuenta dos aspectos: la contribución de la formación recibida respecto al desarrollo de actividades propias de los procesos investigativos y por otra parte, la contribución al desarrollo de competencias investigativas específicas.

5.1. Actividades investigativas. Este aspecto esencialmente se relaciona con el tratamiento de la información que se origina en un proceso investigativo, y que se ven favorecido con el desarrollo de herramientas tecnológicas y servicios informáticos que facilitan el acceso a la información. Para las instituciones educativas de menores recursos, el desarrollo de éstas actividades significa, por una parte, la oportunidad de acceder a gran cantidad de recursos valiosos antes escasos o inexistentes, tales como bibliotecas, laboratorios, mapotecas, hemerotecas, revistas, etc., y por la otra, la necesidad urgente de enseñar a los estudiantes a encontrar esa información, evaluarla de manera crítica y usarla efectivamente. Como acertadamente afirmó el Premio Nobel Herbert Simón, el significado de "saber" ha pasado de poder recordar y repetir información a poder encontrarla y utilizarla (Simón, 1996).

De acuerdo al análisis llevado a cabo, el proceso de desarrollo del proyecto pedagógico de aula se encuentra estrechamente vinculado a las anteriores tareas, permitiendo que la información circule a través de los diferentes espacios y actores de la comunidad educativa. Ello permite que se pueda potencializar en los estudiantes el desarrollo de conocimientos, habilidades y disposiciones personales a partir de la formulación de preguntas que lo acerquen a las diversas fuentes de 
información y le permitan comprender los aportes que ellas hacen, facilitando la adquisición de habilidades para aprender a aprender, construir y compartir saberes, para lo cual será pertinente crear estrategias orientadas a la puesta en práctica de habilidades para identificar la información pertinente, así como las herramientas más adecuadas para combinarlas y convertirlas en conocimientos útiles aplicables en el contexto y en el tema abordado.

Puntualmente, para determinar la actitud de los docentes participantes en este estudio frente al apoyo de las TIC en actividades investigativas, se utilizó una escala tipo Likert que permite analizar los resultados clasificándolos desde actitudes muy favorables en torno al tema de estudio, hasta actitudes muy desfavorables (Baptista, Hernández \& Fernández: 1990).

Los resultados demuestran una actitud favorable frente a la contribución de las TIC para todas las actividades investigativas que se pretendió fomentar con la formación ofrecida: búsqueda de información, selección y clasificación de información, experimentación, tabulación de datos, análisis de datos, elaboración de tablas y gráficos, sistematización y presentación de resultados, acceso y uso de bases de datos, revisión bibliográfica, comunicación con expertos, participación en comunidades, elaboración de informes y elaboración de presentaciones. Esta actitud favorable se puede observar claramente si se tiene en cuenta que el $92 \%$ de los docentes manifiestan estar totalmente de acuerdo o de acuerdo, con la idea de que las TIC estudiadas contribuyen a las actividades investigativas enunciadas. Gráficamente los resultados de la actitud observada se puede representar de la siguiente manera:

Gráfica 1- Actitud de los docentes

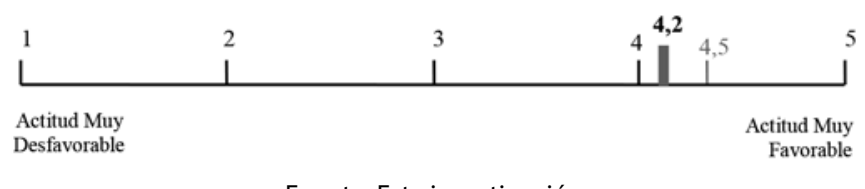

Fuente: Esta investigación

Los resultados también indican que solo existe un 4,3\% de los docentes que manifiestan una actitud desfavorable y se debe principalmente a que en algunos casos las sedes educativas carecen de los recursos necesarios para llevar a cabo las actividades investigativas o realizarlas de la forma adecuada, algunos también necesitan fortalecer la formación de índole pedagógico a fin de clarificar las estrategias didácticas a implementar en el aula, e inclusive, se presentan casos de resistencia al cambio, en los que se hace necesario realizar un proceso motivacional con el docente para generar un cambio de actitud.

5.2. Competencias Investigativas. Más allá de las actividades analizadas, una buena integración de TIC dentro de procesos investigativos se ve reflejada en el desarrollo de las competencias específicas que hacen parte de la investigación, es decir todos aquellos conocimientos, actitudes y habilidades que favorecen el desarrollo de las actividades asociadas al análisis de un problema del contexto, el 
planteamiento de soluciones y su abordaje desde el campo investigativo; lo cual, en este caso se traduce en el planteamiento, ejecución y evaluación del proyecto pedagógico de aula.

Para el análisis, se toma como punto de partida la certeza que en el sector educativo, la investigación hace parte del rol del docente, ya que es un proceso inherente a la enseñanza y el aprendizaje. En el análisis correspondiente se realizó una clasificación de competencias para investigar que pueden ser abordadas y trabajadas por los docentes y la medida en que las TIC contribuyen a potencializar tales habilidades en situaciones y actividades propias del acto investigativo. La siguiente tabla describe los resultados encontrados:

Tabla 1. Contribución de las TIC al desarrollo de competencias investigativas

\begin{tabular}{|l|c|c|c|c|c|}
\hline \multicolumn{5}{|c|}{ Las TIC contribuyen al desarrollo de las COMPETENCIAS INVESTIGATIVAS necesarias para: } \\
\hline \multicolumn{1}{|c|}{ COMPTENCIAS } & TA & DA & NAD & D & TD \\
\hline Observar la realidad & $35,2 \%$ & $52,8 \%$ & $7,8 \%$ & $1,3 \%$ & $3,3 \%$ \\
\hline Descubrir la realidad & $25,4 \%$ & $57,2 \%$ & $12,0 \%$ & $1,9 \%$ & $3,8 \%$ \\
\hline Explicar la realidad & $29,1 \%$ & $57,9 \%$ & $8,4 \%$ & $1,5 \%$ & $3,4 \%$ \\
\hline Predecir la realidad & $16,1 \%$ & $43,8 \%$ & $25,2 \%$ & $10,9 \%$ & $4,4 \%$ \\
\hline Problematizar la realidad & $21,4 \%$ & $51,8 \%$ & $14,7 \%$ & $8,6 \%$ & $3,8 \%$ \\
\hline Generar preguntas de investigación & $37,1 \%$ & $55,4 \%$ & $3,4 \%$ & $0,8 \%$ & $3,6 \%$ \\
\hline Proponer soluciones & $35,6 \%$ & $56,4 \%$ & $3,3 \%$ & $0,6 \%$ & $4,6 \%$ \\
\hline Implementar soluciones & $38,0 \%$ & $54,1 \%$ & $4,0 \%$ & $0,8 \%$ & $3,4 \%$ \\
\hline Generar conocimiento & $47,4 \%$ & $46,1 \%$ & $2,3 \%$ & $0,2 \%$ & $4,4 \%$ \\
\hline Debatir conocimiento con expertos & $39,2 \%$ & $50,5 \%$ & $7,1 \%$ & $1,0 \%$ & $2,7 \%$ \\
\hline Analizar resultados investigativos & $41,3 \%$ & $51,6 \%$ & $2,9 \%$ & $1,7 \%$ & $2,9 \%$ \\
\hline Desarrollar pensamiento creativo & $42,1 \%$ & $49,7 \%$ & $4,6 \%$ & $1,5 \%$ & $2,5 \%$ \\
\hline Implementar metodologías propias & $37,7 \%$ & $53,9 \%$ & $4,4 \%$ & $1,0 \%$ & $3,4 \%$ \\
\hline Compartir resultados investigativos & $46,5 \%$ & $48,2 \%$ & $1,3 \%$ & $0,8 \%$ & $3,6 \%$ \\
\hline PROMEDIO & $35,1 \%$ & $\mathbf{5 2 , 1} \%$ & $\mathbf{7 , 3} \%$ & $\mathbf{2 , 3} \%$ & $\mathbf{3} \% \%$ \\
\hline $\begin{array}{l}\text { TA: Totalmente de acuerdo, DA: De acuerdo, NAD: Ni de acuerdo, ni en desacuerdo, D: En desacuer- } \\
\text { do, TD: Totalmente en desacuerdo. }\end{array}$ & & & \\
\hline
\end{tabular}

Fuente: Esta investigación

Las competencias específicas que tienen una mejor valoración se relacionan con la oportunidad que encuentra el docente para descubrir la realidad, explicarla, generar preguntas de investigación e implementar soluciones a las problemáticas 
abordadas. En el extremo contrario (menor favorabilidad) se encuentran el predecir y problematizar la realidad, las cuales se ven más asociadas al trabajo intelectual que realiza el investigador y que se podría asumir, guarda poca relación con las labores técnicas que son más cercanas al uso de TIC. En promedio, la tendencia de la percepción es favorable y se encuentra representada en la siguiente gráfica:

\section{Gráfica 2: Oportunidad del docente para descubrir la realidad}

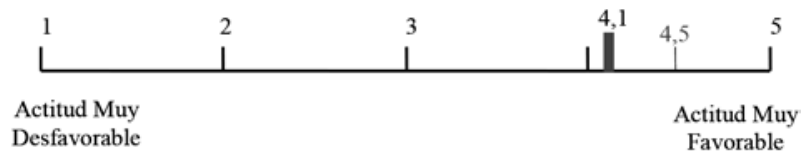

Fuente: Esta investigación

La favorabilidad otorgada a estos aspectos viene determinada por el potencial que tiene la investigación en los procesos educativos y bajo la necesidad de establecerla como eje transversal que articula la teoría con la práctica en un encuentro de saberes entre el profesor y el estudiante. El docente es consciente que para satisfacer esta necesidad y contribuir con el cambio social, se hace indispensable realizar una revisión de las prácticas y las metodologías para abordar el aprendizaje, particularmente en el fortalecimiento de competencias para investigar. Al respecto señala Bachelard (citado por Ugas, 2005) que en el acto de enseñar el profesor no sólo enseña contenidos sino también una manera de pensar el mundo; lo cual devela que los estudiantes aprenden una forma de observar la realidad, analizarla y reelaborarla en la forma proyectada por el profesor.

Los beneficios que otorgan las TIC sobre las competencias investigativas y que genera una actitud de mayor favorabilidad, se ve evidenciado en la generación de conocimientos, ya que el 93,5\% de los docentes manifestó estar totalmente de acuerdo o de acuerdo con el apoyo de las TIC a dicha competencia.

Efectivamente, las acciones relacionadas con observar, explicar, descubrir, problematizar y predecir la realidad tienen una connotación de complejidad más amplia, pues se relacionan con acciones que re-significan el conocimiento; sin embargo, éstas pueden ser adaptadas al contexto educativo y reforzadas con las actividades de investigación realizadas para llegar a los resultados y conclusiones correspondientes.

5.3. Factores asociados al mejoramiento de competencias investigativas. Para estudiar este punto, se analizó cuáles de los aspectos relacionados con el uso de las TIC se constituyen en ventajas o desventajas para el desarrollo de competencias investigativas en el entorno escolar. Los resultados pueden ser observados en la siguiente tabla: 
Tabla 2. Factores asociados al mejoramiento de competencias investigativas

\begin{tabular}{|l|c|c|}
\hline \multicolumn{1}{|c|}{ Factores } & Ventaja & Desventaja \\
\hline Capacitación en TIC de docentes & $91,6 \%$ & $8,4 \%$ \\
\hline Situación del entorno & $88,0 \%$ & $12,0 \%$ \\
\hline Adaptación al uso de TIC & $85,3 \%$ & $14,7 \%$ \\
\hline Cantidad de recursos tecnológicos disponibles & $78,9 \%$ & $21,1 \%$ \\
\hline Disponibilidad de conexión a Internet & $63,8 \%$ & $36,2 \%$ \\
\hline Conocimientos en TIC por parte de estudiantes & $56,2 \%$ & $43,8 \%$ \\
\hline
\end{tabular}

Fuente: Esta investigación

De acuerdo a los resultados, la principal ventaja radica en la capacitación en TIC de los docentes, especialmente la ofrecida con el propósito de lograr su apropiación pedagógica, lo cual permite que puedan desarrollar sus propias competencias y replicar dicha cualificación con sus estudiantes.

Entre los aspectos que brindan ventajas para la investigación educativa mediada por TIC, dos elementos que vale la pena resaltar son la situación del entorno y la adaptación al uso de TIC (88\% y 85\% de valoración positiva respectivamente). En contraste con los resultados respecto a los aspectos favorables analizados, las principales dificultades encontradas se relacionan con el difícil acceso y poca disponibilidad de internet en las instituciones educativas $(36,2 \%$ de los docentes han manifestado esta desventaja) y los escasos conocimientos en TIC por parte de los estudiantes $(43,8 \%)$.

Con respecto a estas últimas dificultades, se destaca sin duda la necesidad imperiosa de contar con el acceso a internet en las instituciones, pues la web no solo se constituye en el más grande repositorio de información académica, sino también en una oportunidad para facilitar la publicación de las experiencias, propuestas y producciones de los mismos docentes. A propósito, según las reflexiones de Sáenz, "la ciberpedagogía es un territorio de información, un mar de información, por donde navega un nuevo pueblo, los investigadores de la educación, navegantes virtuales que intercambian conocimiento de costa a costa” (Sáenz, 1998: 93). Pese a lo anterior, los resultados de esta investigación demuestran que persiste un problema en algunas de las sedes educativas beneficiadas por la estrategia, las cuales por sus características geográficas, aún no pueden contar completamente con el beneficio de conectividad.

Ahora bien, aunque este punto se constituye en una de las principales desventajas encontradas por el $36.2 \%$ de los docentes encuestados dentro de sus instituciones, el potencial de internet ya es reconocido por los docentes y forma parte de las herramientas usadas por ellos en las prácticas académicas e investigativas; esto a pesar de no contar con la infraestructura tecnológica necesaria para obtener el mayor provecho. Ante esta situación, es muy importante que las instituciones educativas estén en disposición de atender esta necesidad, gestionando los recursos necesarios para lograr conectividad deseada. 
Respecto a la dificultad que representa los escasos conocimientos por parte de los estudiantes en herramientas informáticas útiles para el proceso formativo (43,8\% de los docentes reconocen un problema en este punto), es necesario tener en cuenta que las instituciones atendidas por Computadores para Educar que hacen parte de esta investigación están ubicadas en sectores rurales en donde hay un acceso muy precario a la tecnología, con dificultades en la señal de celular, acceso a internet y luz eléctrica, situaciones que influyen en la opinión de los docentes respecto a la existencia de un bajo dominio de las herramientas informáticas por parte de los estudiantes. Dicha situación se constituye en un reto interesante para el sector educativo, ya que a la par de los procesos de incorporación de tecnología educativa, también se requiere avanzar en los procesos de alfabetización digital que le permitan a los estudiantes disfrutar de diferentes beneficios que les ofrecen las modernas tecnologías de información y comunicación.

\section{Discusión}

Es urgente emprender la investigación y los experimentos educativos que traten de superar con ahínco ese inmenso vacío que deja la educación académica, alejada radicalmente de la problemática social de los más pobres. José María Vélaz (Citado por Cendales \& Mariño, 2003, p. 5).

La influencia de las tecnologías de la información y la comunicación es incuestionable y hoy en día forman parte de la cultura tecnológica que nos rodea y con la que debemos convivir. Diversos estudios, opiniones, artículos e investigaciones así lo confirman y continúan, día a día, intentando rescatar todas las oportunidades y beneficios que ofrecen. Este panorama requiere que se reorienten las necesidades de formación de los estudiantes para cumplir con los requerimientos y retos de esta nueva generación, ya que ellos difieren sustancialmente a las que existieron hace algunas décadas. Para lograrlo, se requiere personas con habilidades de autonomía, competitividad, autoaprendizaje, adaptabilidad al nuevo rumbo tecnológico; es decir, competencias (conocimientos, habilidades y actitudes) que son requeridas para dar solución a las problemáticas que se presentan cada día (Tobón, Pimienta \& García, 2010).

En este nuevo escenario, la educación ha cambiado tratando de no desviarse de su propósito fundamental de buscar siempre la formación integral de los niños y jóvenes, a través de un proceso que responda a las necesidades de una comunidad y una sociedad cambiante y demandante. A esto se suma una creciente necesidad de ciudadanos calificados que sepan enfrentarse a un ambiente competitivo y cuenten con las competencias necesarias para enfrentarse a la toma de decisiones, a la obsolescencia rápida de muchos conocimientos, a la rapidez de los cambios, al desarrollo tecnológico, y a la necesidad de construcción y reconstrucción del conocimiento a partir de la investigación.

La investigación, precisamente, es un acto humano intencional que se realiza para construir conocimientos que son utilizados por el hombre para intervenir su 
entorno. La educación y la investigación están íntimamente relacionadas puesto que a partir de ésta relación se pueden obtener resultados exitosos en diversas áreas. Esta es la premisa, y el eje fundamental de la formación ofrecida por la Universidad de Nariño y Computadores para Educar, en el proceso de apropiación pedagógica de TIC.

En dicha formación, en primer lugar se fomentan las habilidades asociadas a identificar, formular y resolver problemas en contextos reales. Para éste caso es indispensable que el docente identifique las situaciones problémicas al interior del aula y evalúe su viabilidad para su abordaje desde un proyecto pedagógico. Para lograrlo será necesario que durante el planteamiento del problema se realice una delimitación coherente con las necesidades educativas de los estudiantes y de las posibilidades de solución y los recursos que ofrece el contexto.

A partir de lo anterior, es necesario que se emprenda el camino de generación de soluciones y alternativas adecuadas para abordar la problemática. El impacto que se logre con la solución propuesta debe ser asumido por el docente, de modo que se valore la retroalimentación y las oportunidades de mejoramiento que se generen a partir de los aportes provenientes de parte de los colegas, directivos, estudiantes y comunidad en general.

La medida del impacto generado en la comunidad educativa con el desarrollo del proyecto, se debe relacionar en buena forma con el uso de tecnología educativa dentro de espacios en los cuales el uso se había restringido a la realización de algunas prácticas mecánicas que no redundan en el aprendizaje de los estudiantes, es decir, que no les permitían adquirir mayores habilidades que las que ofrece el dispositivo o el software como tal. La implementación de TIC para la construcción de los resultados de la investigación debe buscar que los estudiantes mantengan un compromiso constante, y que busquen que las evidencias del proceso, elaboradas y sistematizadas por ellos mismos, resulten atractivas a los ojos de la comunidad. Aquí el docente debe desempeñar un papel fundamental, pues además de conducir el proceso metodológico que conlleva el desarrollo del proyecto de aula, debe crear las actividades que lleven a la consecución del objetivo, realizando una inclusión coherente de las herramientas adecuadas, lo que indica que además deberá tener un buen manejo de la herramienta y sus usos.

Otro de los aspectos a fortalecer, se encuentra ligado a la necesidad de que los resultados que se derivan de la ejecución de los proyectos pedagógicos de aula deben ser dados a conocer a toda la comunidad académica, a fin de obtener los correctivos del proceso, generar alternativas de fortalecimiento y proyectar una nueva forma de construir conocimiento. Es aquí donde docentes y estudiantes pueden confluir en la utilización de los medios tecnológicos de comunicación, el intercambio de saberes a través de las redes y la discusión, dado que en muchos contextos e instituciones educativas se desconocen las iniciativas docentes, coartando la posibilidad de crear y dar sostenibilidad a los proyectos.

Dentro de todo el proceso analizado, es importante que la investigación se convierta en un hábito para docentes y estudiantes, y en el eje transversal del currículo de las instituciones educativas. En reiteradas ocasiones se ha manifestado la necesidad de trabajar con la investigación como eje transversal en el currículo 
institucional y adicionalmente, convertirlo en un objetivo misional de las instituciones educativas. Dicho proceso debe iniciar promoviendo ese enfoque investigativo dentro del trabajo de las instituciones educativas y al interior del aula de clases, promoviendo el desarrollo de actividades que generen espacios de investigación y que propicien la creación de pequeños proyectos; en este sentido, el papel inicial que cumple el docente al interior del aula de clase se debe convierte en el eje fundamental para la investigación.

Finalmente, con el análisis realizado en la presente investigación sobre las prácticas realizadas por los docentes y de la intervención generada en el aula, se inicia la contribución formal hacia al logro de los objetivos en investigación con los estudiantes, lo cual debe iniciar con la revisión de las competencias que se necesitan para investigar y que se deben trabajar al interior de aula. El propósito es que se facilite la construcción de conocimientos y que se brinde a los estudiantes la oportunidad de que sean protagonistas de su propio aprendizaje; así se logrará avanzar a una concepción más completa de formación integral en la que la investigación se constituya en uno de los pilares fundamentales en la educación del siglo XXI.

\section{Conclusiones}

Una de las propuestas más reiteradas en las discusiones educativas en innovación pedagógica es el planteamiento de una dinámica investigativa al interior del aula de clase, situación de la cual surge la presente investigación.

En el marco de la formación ofrecida por Computadores para Educar y recibida por los docentes participantes de este estudio, dicha dinámica investigativa se planteó con el fin de organizar las actividades del proceso de enseñanza y aprendizaje en función a los asuntos que despiertan el interés de los estudiantes, incorporando interrogantes y problemáticas reales que reten su intelecto, su creatividad y que los involucre activamente como protagonistas de su propio proceso de aprendizaje.

Ahora bien, pese a que la investigación pedagógica es considerada como una de las estrategias de enseñanza y aprendizaje mejor referenciada y de obligatorio abordaje en la actualización del sistema educativo, se ha observado que su implementación dentro del aula como eje estructural del currículo sigue siendo escasa o superficial, circunscrita esencialmente a grupos de docentes interesados por cambiar sus prácticas educativas. Pese a esto, en la actual era digital las posibilidades de este enfoque parecen acrecentarse y se han desarrollado diferentes propuestas de utilización de dispositivos digitales para un tratamiento del currículo basado en la investigación escolar. Dentro de ese enfoque se ha desarrollado el proceso formativo promovido por Computadores para Educar y analizado en el presente artículo. Alrededor de ello existen distintos enfoques y diseños metodológicos que permiten organizar la práctica educativa a partir de proyectos investigativos que tienen sus ejes fundamentales en la investigación escolar y la implementación de las TIC.

La estrategia de formación y acceso para la apropiación pedagógica de las TIC que fue analizada, ofrece un enfoque particular hacia el fortalecimiento de las competencias investigativas en los docentes mediante el desarrollo de un proyecto pedagógico de aula que incorpora el uso de tecnología educativa y herramientas TIC para dar solución a una problemática del contexto (Guzmán \& Paz, 2013). Si bien la 
formación se otorga inicialmente al profesorado, se busca que el tratamiento de las competencias investigativas se vea reflejado en forma directa sobre los estudiantes, al verificar que son comprendidas y asimiladas en su participación en el proyecto de aula a través de la puesta en práctica de sus conocimientos en las actividades ejecutadas con ayuda de las TIC.

Volviendo a la formación y actualización docente en la propuesta formativa analizada, se pudo constatar que esta se plantea como un proceso permanente que busca el desarrollo de competencias de diferente tipo: pedagógicas, actitudinales, disciplinares, comunicativas, evaluativas, tecnológicas e investigativas. Esta concepción holística de la labor del docente busca que esté en capacidad de enfrentarse a diferentes retos, orientando en forma adecuada el proceso de formación integral de sus estudiantes.

Específicamente, en el tema propio de este estudio, los docentes favorecidos con la "Estrategia de Formación y Acceso para la Apropiación Pedagógica de las TIC” se han visto beneficiados con la posibilidad de fortalecer el desarrollo de sus competencias investigativas a partir de la formación recibida, lo cual se complementa durante el planteamiento, ejecución y evaluación de su proyecto pedagógico aula; sin embargo este proceso formativo y de desarrollo de su proyecto, ha permitido poner en evidencia que la formación en competencias investigativas de los docentes es muy escasa y requiere ser fortalecida haciendo un énfasis especial en los nuevos paradigmas de investigación y en la integración de TIC dentro de los procesos asociados.

El primer escenario de investigación docente es el aula. En la medida en que el docente investigue y reflexione sobre los hechos que rodean su práctica educativa, puede identificar las competencias que requiere en la investigación, tanto en el plano académico como personal; así mismo, podrá promover la puesta en práctica de estas competencias dentro del contexto inmediato de acción, sin que esto signifique que únicamente el maestro sea el responsable de todo el trabajo investigativo, pero sí que en sus manos se encuentra la responsabilidad de orientar el proceso correctamente.

El fortalecimiento de las competencias investigativas en los docentes permite un logro adicional y es el de estar en capacidad de promover estas mismas competencias en el grupo de estudiantes con quienes adelanta su proyecto de aula. Esto abre la posibilidad de que puedan alcanzar su real potencial en la construcción de sus propios conocimientos, y a su vez, realicen un aporte en la estrategia a implementar para solucionar el problema y lograr los objetivos planteados.

La investigación educativa toma el rumbo de las tendencias de la educación. En éste sentido, teniendo en cuenta que el ritmo educativo está siendo influenciado notoriamente por las TIC, los maestros son elementos fundamentales para que la investigación asuma un nuevo rumbo en el que se incluyan estas nuevas herramientas; así, se logrará avanzar en un doble propósito: afianzar los procesos investigativos y promover la integración curricular de estas tecnologías. Por ello es preciso que los profesores además de adquirir las competencias en investigación, adquieran las habilidades en tecnología para obtener provecho de ella, especialmente en las actividades asociadas a la consolidación y difusión de resultados. 
Si bien los beneficios parecen claros, una de las dificultades fundamentales a las cuales se enfrentan las instituciones educativas analizadas es lograr la integración de la investigación como estrategia para el mejoramiento del aprendizaje, incluyendo la utilización de las TIC como una de sus principales herramientas. Para ello, es necesario que se haga una articulación de niveles, áreas y contenidos, en los que se propicien los escenarios de acción cooperativa entre los distintos actores del proceso formativo, lo cual finalmente redundará en beneficio de los estudiantes. Los resultados de la formación a docentes, del desarrollo de proyectos de aula y de la presente investigación respecto al desarrollo de competencias investigativas, dan cuenta de este potencial y de las enormes posibilidades de mejoramiento de la educación a partir de este tipo de estrategias.

\section{REFERENCIAS}

1. CASTELLS, Manuel (1997). La era de la información, economía, sociedad y cultura. Volumen 1, La Sociedad Red, Alianza Editorial, Madrid, p. 174.

2. CENDALES, Lola; MARIÑO, Germán (2003). Aprender a Investigar investigando, Colección“Programa Internacional de Formación de Educadores Populares", Fundación Santa María, Venezuela. Recuperado de: http://www.feyalegria.org/images/acrobat/Folleto\%2013\%20Aprender\%20a\%20investigar,\%20 investigando_2819.pdf

3. COMPUTADORES PARA EDUCAR (2012). Especificaciones técnicas estrategia de formación y acceso para la apropiación pedagógica de las TIC 2014. Ministerio de Tecnologías de la Información y las Comunicaciones. Bogotá, enero, pp. 3-4.

4. COMPUTADORES PARA EDUCAR (2012). Pliego de condiciones: selección abreviada de menor cuantía. No. 011 de 2012. Ministerio de Tecnologías de la Información y las Comunicaciones. Bogotá, agosto, $207 \mathrm{p}$.

5. DELORS, Jaques (1996). La educación encierra un tesoro. En: Informe a la Unesco de la Comisión Internacional sobre la educación para el siglo XXI, Compendio, Ediciones Unesco, pp. 16-17. Recuperado de: http://www.unesco.org/education/pdf/DELORS_S.PDF.

6. GARDNER, Howard (1998). Inteligencias múltiples: la teoría en la práctica. Paidós Ibérica, Barcelona, $314 \mathrm{p}$.

7. GONZÁLEZ D., Carlos; SÁNCHEZ S. Leonardo (2003). El diseño curricular por competencias en la educación médica. Revista Educación Médica Superior, Escuela Nacional de Salud Pública, Vol. 17, Núm. 4. Recuperado de: http://bvs.sld.cu/revistas/ems/ vol17_4_03/ems04403.htm

8. GONZALEZ M., Viviana (2000). ¿Qué significa ser un profesional competente? Reflexiones desde una perspectiva psicológica. Revista lberoamericana de Educación, Núm. 8, Diciembre, pp. 1-4. Recuperado de: http://www.rieoei.org/deloslectores/Maura.PDF. 
9. GUZMÁN, Claudia; PAZ, Dayra (2013). Apropiación pedagógica de las TIC: Guía de Formación Docente. Computadores para Educar - Universidad de Nariño, Centro de publicaciones, Marzo, pp. 24-42.

10. HELLER, Agnes (1987). Sociología de la vida cotidiana. Editorial Península, Barcelona, pp. 317.

11. HERNÁNDEZ S., Roberto; FERNÁNDEZ C., Carlos; BAPTISTA L., Pilar (1991). Metodología de la Investigación. McGraw Hill, México, pp. 54.

12. Ley 115 de 1994, Por la cual se expide la Ley General de la Educación en Colombia, Diario oficial No. 41.214 de 8 de febrero de 1994.

13. MINISTERIO DE TECNOLOGÍAS DE LA INFORMACIÓN Y LAS COMUNICACIONES (2008). Plan nacional de tecnologías de la información y las comunicaciones. Bogotá, marzo, pp. 3-8.

14. MUÑOZ, José F.; QUINTERO C., Josefina; MUNEVAR M., Raúl (2001). Cómo desarrollar competencias investigativas en educación, Editorial Magisterio, Bogotá, julio, Tercera Edición, pp. 211-224.

15. SÁENZ A., Rafael (1998). La ciberpedagogía y la investigación educativa ante el nuevo milenio. Revista Complutense de Educación, Vol. 9, Num. 1, pp. 92. Recuperado de: http://revistas.ucm.es/index.php/ RCED/article/view/ RCED9898120085A/17393

16. SIMON, H.A. (1996). Observations on the Sciences of Science Learning. Departamento de Psicología, Universidad Carnegie Mellon.

17. TAYLOR S.; BOGDAN, R.C. (1989). Introducción a los métodos cualitativos de investigación. Paidós, Barcelona.

18. TOBÓN T., Sergio; PIMIENTA P., Julio; GARCÍA F., Juan (2010). Secuencias Didácticas: Aprendizaje y Evaluación de Competencias. Recuperado de http://www.urosario.edu.co/CGTIC/Documentos/ secuencias_didacticas.pdf.

19. UGAS, Gabriel (2005). Epistemología de la educación y la pedagogía. Ediciones del Taller de Formación Permanente de Estudios Epistemológicos en Ciencias Sociales, Táchira, $151 \mathrm{p}$.

20. UNESCO (2008). Estándares de Competencia en TIC para docentes, Londres. Recuperado de: http:// www.oei.es/tic/UNESCOEstandaresDocentes.pdf. 